\title{
CMOS microelectrode array for the monitoring of electrogenic cells
}

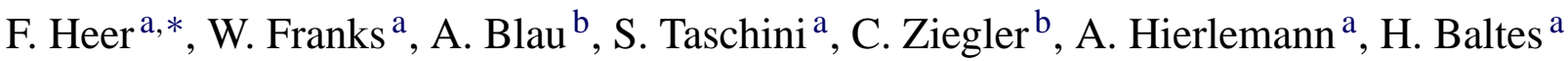 \\ ${ }^{a}$ Physical Electronics Laboratory, ETH Zurich, 8093 Zurich, Switzerland \\ ${ }^{\mathrm{b}}$ Department of Biophysics, University of Kaiserslautern, 67663 Kaiserslautern, Germany
}

Received 21 October 2003; received in revised form 4 February 2004; accepted 5 February 2004

\begin{abstract}
Signal degradation and an array size dictated by the number of available interconnects are the two main limitations inherent to standalone microelectrode arrays (MEAs). A new biochip consisting of an array of microelectrodes with fully-integrated analog and digital circuitry realized in an industrial CMOS process addresses these issues. The device is capable of on-chip signal filtering for improved signal-to-noise ratio (SNR), on-chip analog and digital conversion, and multiplexing, thereby facilitating simultaneous stimulation and recording of electrogenic cell activity. The designed electrode pitch of $250 \mu \mathrm{m}$ significantly limits the space available for circuitry: a repeated unit of circuitry associated with each electrode comprises a stimulation buffer and a bandpass filter for readout. The bandpass filter has corner frequencies of $100 \mathrm{~Hz}$ and $50 \mathrm{kHz}$, and a gain of 1000 . Stimulation voltages are generated from an 8-bit digital signal and converted to an analog signal at a frequency of $120 \mathrm{kHz}$. Functionality of the read-out circuitry is demonstrated by the measurement of cardiomyocyte activity. The microelectrode is realized in a shifted design for flexibility and biocompatibility. Several microelectrode materials (platinum, platinum black and titanium nitride) have been electrically characterized. An equivalent circuit model, where each parameter represents a macroscopic physical quantity contributing to the interface impedance, has been successfully fitted to experimental results.
\end{abstract}

(c) 2004 Elsevier B.V. All rights reserved.

Keywords: Microelectrode array; CMOS; Electrogenic cells; Cardiomyocites; Cell-based biosensor; Impedance

\section{Introduction}

Since its inception in the early 1970s (Wise, 1971; Thomas et al., 1972), the microelectrode array (MEA) has evolved into a tool capable of making significant contributions to the fields of neuroscience and biosensing (Rutten, 2002). The primary advantages of extracellular recordings over traditional methods (patch clamp, voltage-sensitive dyes) include non-invasiveness, multi-site measurement capabilities, and long measurement periods. However, despite concentrated research efforts widespread use has yet to be achieved as crucial design and development issues, such as low signal-to-noise ratio (SNR), flexible stimulation and readout capabilities, perfusion chambers with coupled microfluidics, and controlled growth techniques have yet to be satisfactorily addressed (Borkholder, 1998; Gross et al., 1995).

MEAs, with their ability to measure complex spatiotemporal neural activity, are poised to become a fundamental tool in neuroscience research.

\footnotetext{
* Corresponding author. Tel.: +41-1633-6577; fax: +41-1633-1054.

E-mail address: heer@iqe.phys.ethz.ch (F. Heer).
}

In developing embryos, synchronized burst activity is thought to be important in fine tuning neuronal networks. MEAs have achieved a high degree of success in the study and characterization of this activity (Keefer, 2001a,b; Tscherter et al., 2001; Streit et al., 2001; Jimbo and Robinson, 2000). To study neurological phenomena such as Hebbian learning, the neural networks must be mapped; this could be achieved using neural tissue slices which exhibit significantly reduced cell mobility. Organotypic culturing of rat hippocampal and spinal cord tissue slices on MEAs (Egert et al., 1998; Thiebaud et al., 1999; Borkholder et al., 1997) demonstrate this potential. Other ways to create defined neural networks are to control the growth of processes with columnar structures (Zeck and Fromherz, 2001), with chemical cues (Prinz and Fromherz, 2000), or to trap the cells in cages (Maher et al., 1999). Shahaf and Marom have shown that learning in cortical neural networks occurs in vitro, thereby demonstrating the effectiveness of MEAs in the study of synaptic plasticity (Shahaf and Marom, 2001; Marom and Shahaf, 2002).

Cell-based biosensors are promising tools for the detection of chemical and biological toxins or for the screening of pharmacologically active compounds (the reader is directed 
to numerous reviews: Kovacs, 2003; McFadden, 2002; Pancrazio et al., 1999; Gross et al., 1995). These systems employ highly evolved cellular pathways and have the advantage that no previous knowledge of the investigated compound is required. Field-portable systems using cardiomyocytes have been developed at the Standford Transducers Lab and the Naval Research Laboratory (DeBusschere and Kovacs, 2001; Gilchrist et al., 2001; Pancrazio et al., 1998b). Neuronal network-based biosensors have been used to demonstrate that in vitro cultures retain tissue-specific properties, and moreover, findings corroborate well with whole animal in vivo studies (Morefield et al., 2000; Keefer, 2001a,b). It can be envisioned that cell-based biosensors will replace animals in toxicology studies (van der Schalie et al., 1999).

The accurate measurement of low extracellular bioelectric signals, ranging from microvolts for neurons to millivolts for cardiomyocytes, at multiple points within a culture are the two key requirements considered when designing an MEA and recording system. Typical system designs incorporate an MEA with an external, off-chip system controlling electrode selection, signal amplification and interfacing (Gross, 1979; Gross et al., 1993; Borkholder, 1998; Connolly et al., 1990; Bucher et al., 1999; Heuschkel et al., 2002; Multi Channel Systems, 2001; Jenkner et al., 2001). However, as the recorded signal travels to the amplification system, passing several electronic interfaces, it can be distorted by electromagnetic interference resulting in a reduced signal-to-noise ratio. A crucial design consideration for large arrays is multiplexing. If multiplexing circuitry is not included each electrode requires an associated bond pad thereby limiting the array size to the number of available bond pads. CMOS integration can overcome both of these technical challenges. First approaches include a two-chip solution, where a CMOS chip with instrumentation amplifiers and electrode selection circuitry is placed in close proximity to the MEA chip (Pancrazio et al., 1998a). Single-chip solutions with fully-integrated CMOS multiplexing and buffering circuitry have been presented, with on-chip (Bai and Wise, 2001) and off-chip (DeBusschere and Kovacs, 2001) signal amplification. More recently systems have emerged, such as a FET-based, 16,384 microelectrode CMOS biosensor array comprising on-chip amplification circuitry with readout multiplexers and buffers (Eversmann et al., 2003), or, similarly, an MEA with on-chip amplification, filtering and addressability (Berdondini et al., 2002). While these designs represent significant improvements in neurochip development and the use of multiplexers translates into almost unconstricted array sizes, they do not yet include flexible on-chip stimulation capabilities.

Here, we report on a new biochip comprising a microelectrode array and fully-integrated analog and digital CMOS circuitry for the stimulation and recording of activity from electrogenic cells. In contrast to the systems mentioned previously, this device is capable of on-chip signal filtering for improved SNR, on-chip analog and digital conversion to prevent signal degradation, multiplexing, and simultaneous recording and stimulation. Each electrode has an associated circuitry unit including a stimulation buffer and switch, a bandpass filter and a readout buffer. The electrode and circuitry form a repeatable unit with a pre-defined $250 \mu \mathrm{m}$ pitch that significantly limits the area available for circuitry. To achieve the electrode pitch, only the components necessary to condition the small signals and to provide reliable, addressable stimulation signals have been assembled in a repeated circuitry unit. This repeatable unit can be multiplied to form a larger array. The stimulation buffer uses a class-AB output stage in order to deliver large currents to the electrode. Stimulation voltages are generated from an 8-bit digital signal converted to an analog signal at a frequency of $120 \mathrm{kHz}$. As a result, any stimulation waveform can be generated. The bandpass filter has corner frequencies of $100 \mathrm{~Hz}$ and $50 \mathrm{kHz}$ and a gain of 1000 . The microelectrode is realized in a shifted design for flexibility and biocompatibility purposes. Several biomedically relevant microelectrode materials, platinum, platinum black and titanium nitride, have been electrically characterized, and an equivalent circuit model of the impedance is presented.

\section{Methods}

\subsection{System design}

The $4.4 \mathrm{~mm} \times 4.4 \mathrm{~mm}$ chip presented here consists of a $4 \times 4$ electrode array with integrated reference electrode (Fig. 1) (Franks et al., 2003). Fabrication was performed using an industrial double-polysilicon, triple-metal, $0.6 \mu \mathrm{m}$ CMOS process at Austriamicrosystems AG (Austria). The electrodes are $40 \mu \mathrm{m} \times 40 \mu \mathrm{m}$ with a $250 \mu \mathrm{m}$ pitch. Space between the electrode array and bond pads has been intentionally left blank for the sealant that isolates the cells from the bond wires. A block diagram with the main circuitry components is given in Fig. 1. A unit of repeating circuitry comprising a buffer and switch for stimulation, a bandpass filter and buffer for readout, is provided with each electrode (inset, Fig. 1). Additionally, the system architecture incorporates four analog-to-digital converters (ADC, one per row of electrodes) and one digital-to-analog converter (DAC). On-chip multiplexing and A/D-conversion allow for a reduction in the number of bondpads, which facilitates the integration of a large array. A digital control unit scans the array during readout, provides individual electrode addressability for stimulation, and interfaces with the outside world. Simultaneous recording and stimulation at each electrode can be performed. For the purpose of monitoring the chip operation temperature and the culture temperature, a temperature sensor has been included.

\subsection{Electrode design}

Due to a limited selection of materials available in a commercial CMOS process, the electrode material as received 

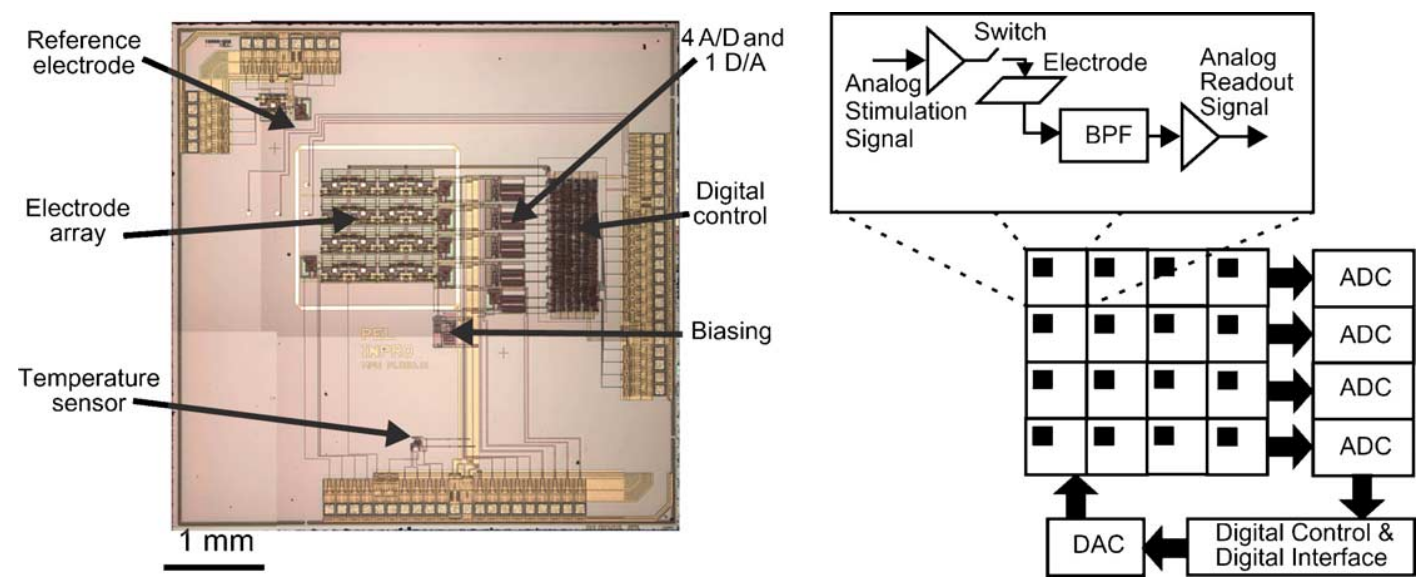

Fig. 1. Photograph of CMOS chip on the left. Block diagram of the system architecture on the right. Inset shows the contents of the repeated circuitry unit.

from the foundry is aluminum, a known neurotoxicant. A simple 2-mask post-processing procedure is employed to cover the $\mathrm{Al}$ electrodes with biocompatible platinum. During this processing the electrodes are shifted (Fig. 2), and the aluminum is sealed with the electrode metal and a passivation stack. It is necessary to seal the underlying Al to avoid both poisoning and undesirable electrochemistry that occurs when the $\mathrm{Al}$ comes into contact with the neuron medium. The electrode metal consists of $50 \mathrm{~nm}$ TiW, an adhesion promoter, and $270 \mathrm{~nm}$ of Pt. The metals are sputter-deposited and structured in a lift-off process. An alternating $\mathrm{Si}_{3} \mathrm{~N}_{4} / \mathrm{SiO}_{2}$ passivation stack consisting of $1 \mu \mathrm{m}$ of $\mathrm{Si}_{3} \mathrm{~N}_{4}$, and two times $100 \mathrm{~nm} \mathrm{SiO}{ }_{2}$ and $200 \mathrm{~nm}$ $\mathrm{Si}_{3} \mathrm{~N}_{4}$ (total layer thickness of $1.6 \mu \mathrm{m}$ ) is deposited using plasma-enhanced chemical vapor deposition (PECVD). A mixed frequency PECVD process was used to match the

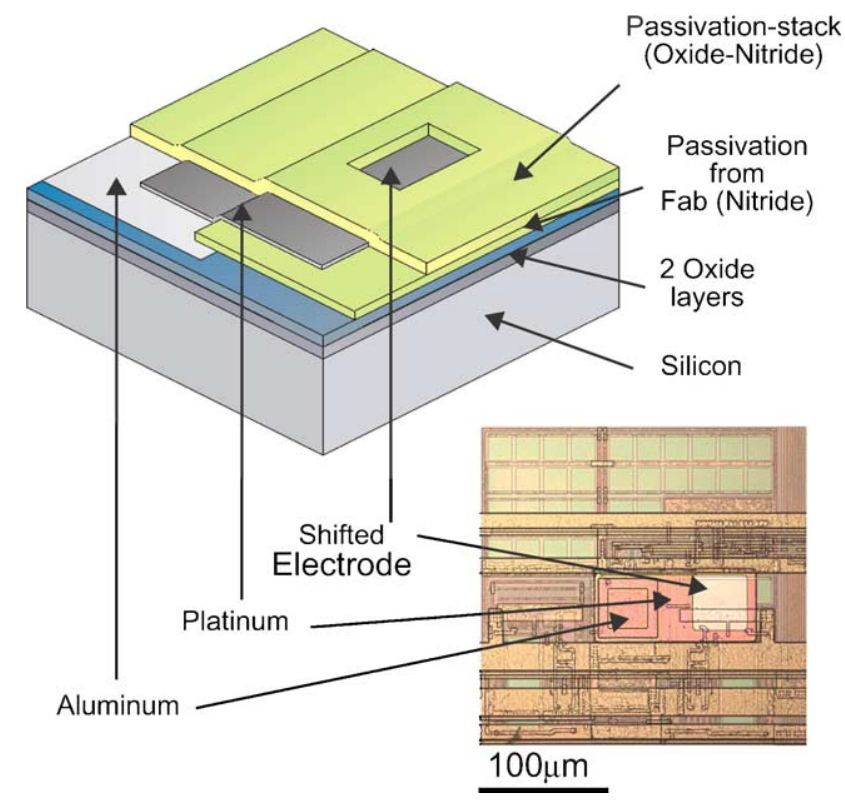

Fig. 2. Cross-section (left) and micrograph (right) of the shifted Pt-electrode. Micrograph shows one circuitry repeating unit. stress with that of the underlying $\mathrm{Si}_{3} \mathrm{~N}_{4}$ deposited during the CMOS process. A simpler solution featuring a single $1 \mu \mathrm{m} \mathrm{Si}{ }_{3} \mathrm{~N}_{4}$ layer has been attempted, but proved to be ineffective in sealing the $\mathrm{Al}$, resulting in the dissolution of the reference electrode during cell culturing. It is believed that the roughness of the underlying Al layer leads to pinholes in the $\mathrm{Si}_{3} \mathrm{~N}_{4}$ layer; however, when a stack of alternating oxide and nitride layers is deposited these pinholes are covered. Neurons have been cultured for 28 days on chips with the $1.6 \mu \mathrm{m}$ passivation stack, and no degradation effects have been observed. A reactive-ion etch (RIE) is then used to open the nitride stack defining the size and shape of the electrodes. This increases design flexibility since the electrode dimensions are not defined by the CMOS process. The processed chips are bonded and packaged in a two-step process. An epoxy (Masterbond 42HT-T, Masterbond Inc., USA) is first used to stabilize the bonds on the chip and the package. Polydimethylsiloxane (PDMS) is then used to form a bath, isolating the bond wires from the cells. A glass O-ring, affixed to the chip with PDMS, forms a larger bath capable of containing a suitable amount of neuron medium (Fig. 3).

\subsection{Electrode characterization and modeling}

Extracellular signals are typically minute making a low electrode-electrolyte interface impedance imperative. To characterize the electrode-electrolyte interface electrochemical impedance spectroscopy (EIS) has been performed for bright Pt, Pt black and TiN. The EIS measurements have been used to model the interface with an equivalent circuit model where each parameter represents a macroscopic physical process contributing to the interface impedance. An enhanced understanding of the physical processes can be used to better understand the transduction principle and to design a lower-impedance electrode.

The measurements have been fitted to the equivalent circuit model (Fig. 4) comprising a constant phase angle impedance, $Z_{\mathrm{CPA}}$, shunted by a charge transfer resistance, 


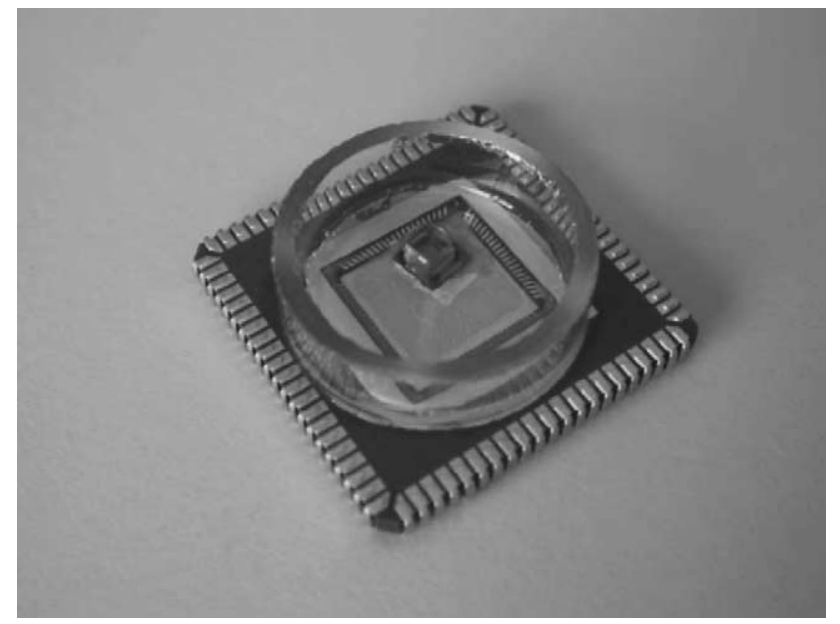

Fig. 3. Image of packaged chip.

$R_{\mathrm{ct}}$, together in series with the solution resistance, $R_{\mathrm{S}}$ (Grahame, 1952). The constant phase angle impedance first proposed by Fricke (1932) is a measure of the non-Faradaic impedance arising from the interface capacitance, or polarization capacitance, and is given by the empirical relation:

$Z_{\mathrm{CPA}}(\omega)=\frac{1}{(j \omega Q)^{n}}$

where $Q$ is a measure of the magnitude of $Z_{\mathrm{CPA}}, n$ is a constant $(0 \leq n \leq 1)$ representing inhomogeneities in the surface and $\omega=2 \pi f$. In a Nyquist plot, the angle between the data and the abscissa axis gives $n$ from $n=(2 \theta) / \pi$. When $n=1, Z_{\mathrm{CPA}}$ represents a pure capacitive element corresponding to the interface capacitance. Faradaic charge transfer leads to $R_{\mathrm{ct}}$, which in the case of $\mathrm{Pt}$ is believed to be due to the hydrolysis of $\mathrm{H}_{2} \mathrm{O}$ and reduction of $\mathrm{O}_{2}$ according to $2 \mathrm{H}_{2} \mathrm{O} \leftrightarrow \mathrm{O}_{2}+4 \mathrm{H}^{+}+4 \mathrm{e}^{-}$where the equilibrium potential is $0.987-0.059 \times \mathrm{pH}$ with respect to the standard calomel electrode (SCE) (Yeager, 1984). Experiment findings support this assumption. When the open-circuit potential (OCP) is set to value higher or lower than the equilibrium value of $\sim 0.6 \mathrm{~V}$, then the $R_{\mathrm{ct}}$ is lower than if the OCP were equal to the equilibrium value. The higher or lower potential shifts the reaction from equilibrium conditions, more Faradaic charge transfer occurs, and $R_{\mathrm{ct}}$ is reduced. For example, for an OCP of $0.66 \mathrm{~V}$ the change transfer resistance is $1.0 \mathrm{M} \Omega$. For an

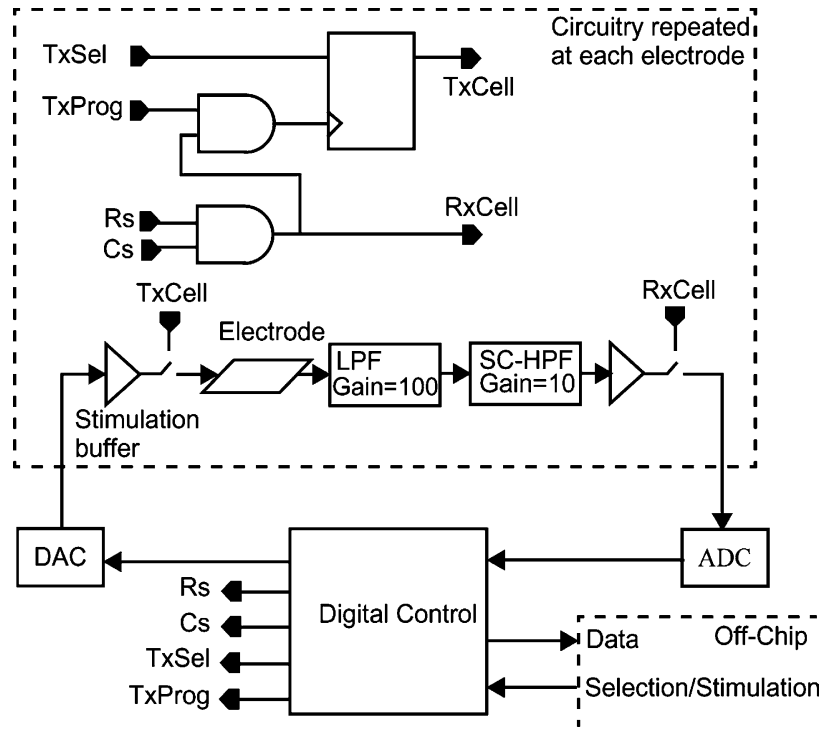

Fig. 5. Schematic of stimulation and readout circuitry.

OCP of 0.39 and $0.92 \mathrm{~V}$ the charge transfer resistance reduces to 0.43 and $0.23 \mathrm{M} \Omega$, respectively. However, it should be noted that the presence of any impurities at the interface will also lead to a reduced charge transfer resistance.

\subsection{Stimulation circuitry}

The chip presented in this paper is capable of stimulating any subset of electrodes with any arbitrary waveform. When a stimulation phase is enabled, the on-chip digital control sets the selection by adjusting the switch between the stimulation-buffer and the electrode (Fig. 5). Since the impedance of the electrode varies significantly with electrode material, size, and electrolyte, a buffer with a class-AB output stage was used in order to be capable of delivering large currents (up to $10 \mathrm{~mA}$ if needed). The buffer (Fig. 6) comprises a differential input stage (M1, M2) with an active load (M3) and is connected in unity gain ( $V_{\text {OUT }}$ connected to gate of M2). The class-AB stage is formed by the transistors M5, M6, M9 and M10, where M5 and M6, both diode connected, bias the output transistors M9 and M10. If the input voltage $\left(V_{\mathrm{IN}}\right)$ is increased, M9 forces the output voltage $\left(V_{\text {OUT }}\right)$ to follow. The load current is delivered by

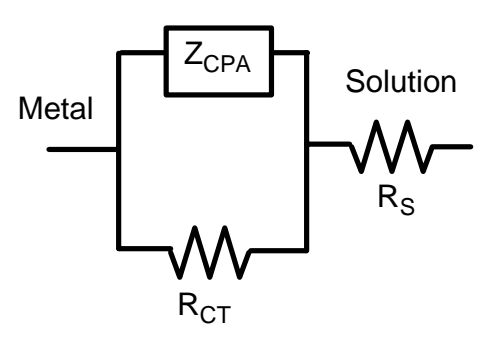

\begin{tabular}{|c|cccc|}
\hline & $\mathrm{Q}$ & $\mathrm{n}$ & $\mathrm{R}_{\mathrm{S}}$ & $\mathrm{R}_{\mathrm{CT}}$ \\
& {$\left[\mathrm{s} \Omega^{-1 / \mathrm{n}}\right]$} & & {$[\Omega]$} & {$\left[10^{5} \Omega\right]$} \\
\hline Pt & $2.72(10)^{-5}$ & 0.92 & 28.0 & 4.48 \\
Std. Dev. & $0.26(10)^{-5}$ & 0.01 & 2.81 & 0.88 \\
\hline Pt Black & $2.08(10)^{-3}$ & 0.91 & 30.7 & 0.51 \\
Std. Dev. & $0.11(10)^{-3}$ & 0.03 & 7.85 & 0.28 \\
\hline TiN & $2.03(10)^{-3}$ & 0.91 & 42.3 & 3.00 \\
Std. Dev. & $5.77(10)^{-5}$ & 0.00 & 3.70 & 3.20 \\
\hline
\end{tabular}

Fig. 4. Equivalent circuit model of electrode-electrolyte interface. Measured results are given in the table. 


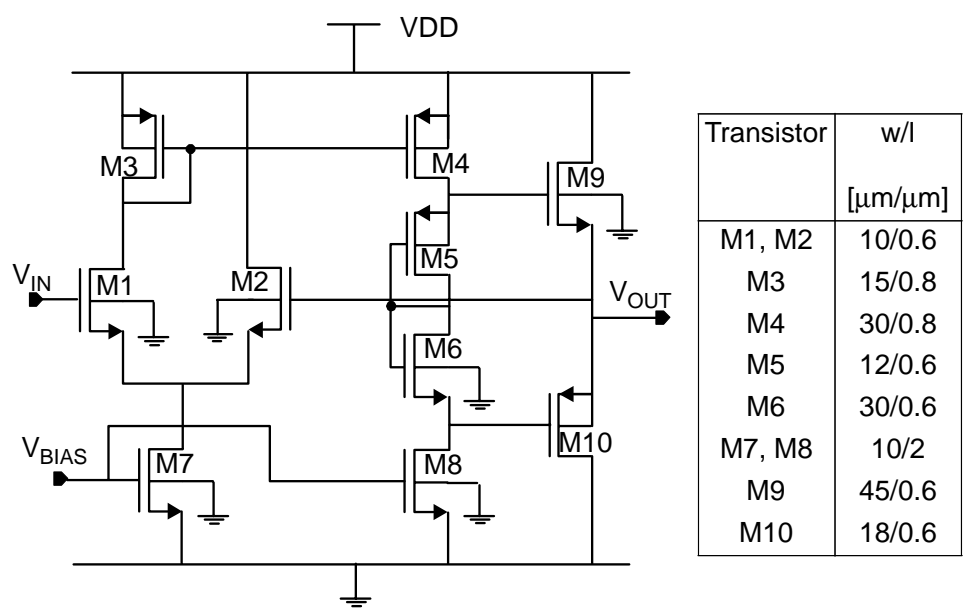

Fig. 6. Schematic of the class-AB stimulation buffer. Transistor sizes are summarized in the table.

M9, whose gate-source voltage ( $\left.V_{\mathrm{GS}}\right)$ will increase slightly. Since a constant voltage is maintained between the gates of M9 and M10 the $V_{\mathrm{GS}}$ of M10 decreases by the same amount that the $V_{\mathrm{GS}}$ in M9 increases. M10 stays on, but conducts only a small current. When $V_{\mathrm{IN}}$ is lowered the situation is inverted, with M10 forcing $V_{\text {OUT }}$ to follow and with the current through M9 decreasing. The power consumption is $45 \mu \mathrm{W}$ for vanishing input, given a current of $2 \mu \mathrm{A}$ through M7 and M8, and a supply voltage ( $\left.V_{\mathrm{DD}}\right)$ of $5 \mathrm{~V}$. However, when slewing, the amplifier can deliver up to $10 \mathrm{~mA}$ to the electrode. This corresponds to a maximum current density of $60 \mathrm{~A} / \mathrm{cm}^{2}$. The whole buffer occupies an area of $42 \mu \mathrm{m} \times 32 \mu \mathrm{m}$ and has a bandwidth of $\sim 10 \mathrm{MHz}$.

The programming of the array is described as follows (Fig. 5). Transmission gates are used as switches for stimulation (TxCell) and readout (RxCell). Both are set by a small control unit consisting of a flip-flop and two AND gates. The row and column signals, RS and CS, are used for readout operation. When RxCell is high the signal is sampled by the ADC.

The programming of the array is done with two signals TxSel and TxProg. The on-chip digital control generates these signals based on the pattern information received from the PC. When RxCell goes high while TxProg is high the flip flop, which controls the stimulation switch, can be set with TxSel.

Subsequent to selection digital stimulation patterns are sent to the chip continuously until the stimulation phase is stopped. Stimulation voltages are in a range given by two reference voltages, which have a resolution of 8-bit and a cycling of $120 \mathrm{kHz}$. The time delay between enabling a stimulation phase and first stimulation voltage to reach the electrode is $25 \mu \mathrm{s}$. The time required to go from one subset of electrodes to the next including deselection, selection and time required for a first stimulation voltage to reach the electrodes is $50 \mu \mathrm{s}$. Stimulation phases can take place at any time throughout the measurement and are not limited in number. For deselection of the electrodes the signal TxProg is set high while TxSel is low.

\subsection{Readout circuitry}

Electrogenic cells induce small signals on the electrodes. The range is tens of microvolts for neural signals up to millivolts for heart cells. In order to prevent signal degradation signal conditioning should be implemented as close to the electrode as possible. For this reason, buffering and filtering circuits are adjacent to and repeated at each electrode (Figs. 1 and 5). The available space for the circuitry blocks is limited by the pitch between two electrodes, which is designed to be $250 \mu \mathrm{m}$. It is possible that several cells produce signals on the same electrode. A $20 \mathrm{kHz}$ sampling frequency is adopted so as to be able to resolve signal components originating from different cells.

When an action potential occurs in a cell above the electrode, ions flowing across the cell membrane inducing a charge redistribution on the electrode. The frequency of these signals is expected to be approximately $1 \mathrm{kHz}$. AC-coupling is mandatory for biological signals due to large drifts. Additionally, low pass filtering limits the noise bandwidth and provides anti-aliasing. Consequently, we implemented a bandpass filter with a center frequency around $1 \mathrm{kHz}$. The corner frequencies of the filter were chosen to be $100 \mathrm{~Hz}$ and $50 \mathrm{kHz}$. Similar specification can be found in literature (DeBusschere and Kovacs, 2001; Bai and Wise, 2001). The bandpass filter is divided into two stages. The first step is a low-pass filter, which buffers and amplifies the signals by a factor of 100 . The electrode is directly connected to the non-inverting input of the amplifier, the gate of a MOSFET, which has a high input impedance in CMOS technology. This is similar to other systems, which have successfully acquired signals from electrogenic cells (Fromherz, 2002, DeBusschere and Kovacs, 2001). Signals in the range of tens of microvolts can be measured since the equivalent input noise of the amplifier is below $10 \mu \mathrm{V}_{\mathrm{RMS}}$ over the band $100 \mathrm{~Hz}-100 \mathrm{kHz}$. Very little area $(120 \mu \mathrm{m} \times 70 \mu \mathrm{m})$ is occupied by the amplifier. Furthermore, the amplifier uses an internally compensated OpAmp, which acts as a low-pass filter with a corner frequency of 
$\sim 50 \mathrm{kHz}$. This corner can vary between 20 and $100 \mathrm{kHz}$ due to process parameter variations. The low-pass transfer function in this first stage provides the anti-aliasing, that is mandatory for the switched-capacitor circuit in the following stage. The high-pass filter implemented in the second stage has a gain of 10 and a corner frequency at $100 \mathrm{~Hz}$. A large time constant is needed in the high-pass filter, in order to achieve the low cut-off frequency of $100 \mathrm{~Hz}$. Since the available space is limited, we used a switched capacitor solution. After the second filter, the signal is buffered and sent to the ADC via an analog multiplexer. As multiplexing takes place after signal amplification and filtering, the design is less sensitive to switching and electromagnetic interference. The ADC uses the successive-approximation technique and has a resolution of 8 bits. A positive and a negative reference voltage define the range. Digital values are finally sent to a PC through an 8-bit parallel bus.

\section{Results}

\subsection{Electrode impedance}

Fig. 7 shows the measured (dashed markers) and modeled (solid line) results of the impedance modulus and phase as a function of frequency for three different materials: bright $\mathrm{Pt}$ $(n=4)$, Pt black $(n=4)$, and TiN $(n=3)$. A large geometric surface area, $1 \mathrm{~cm}^{2}$, was initially used to avoid complications arising from low current measurements typical of very small areas. Measurements were performed using a commercially available Autolab PGSTAT30 potentiostat system with Frequency Response Analysis software (version 4.8, Eco
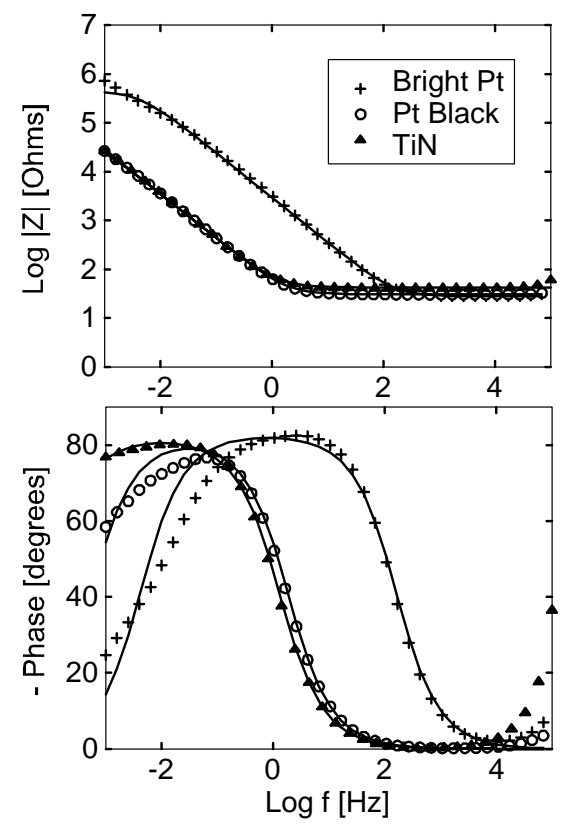

Fig. 7. Independence modulus and phase as a function of frequency for $1 \mathrm{~cm}^{2}$ Pt, Pt-black and TiN electrode materials.
Chemie B.V., Netherlands). In this three electrode system a standard calomel electrode (SCE) is the reference electrode, the counter electrode is a large-area Pt-mesh, and the electrolyte is $0.9 \% \mathrm{NaCl}$. The perturbation potential was $10 \mathrm{mV}$ and the scan range was $10^{-3}$ to $10^{5} \mathrm{~Hz}$. The Pt electrodes were additionally treated by voltammetric cycling from -0.5 to $1.0 \mathrm{~V}$ for typically six cycles, when the measurement stabilized. This procedure potentially results in the formation and reduction of $\mathrm{Pt}$ oxide and $\mathrm{Pt}$ dioxide layers, as indicated by the $\mathrm{Pt}-\mathrm{H}_{2} \mathrm{O}$ Pourbaix diagram (Pourbaix, 1974). Before performing the impedance scan the open-circuit potential (OCP) was recorded. The OCP is a function of the chemical composition of the interface, and can be used for quality control of the initial interface conditions. Average OCP values for Pt, Pt black and TiN were $0.318 \pm 0.037$, $0.299 \pm 0.061$, and $0.046 \pm 0.034 \mathrm{~V}$, respectively.

\subsection{Equivalent circuit model parameter results}

The derived values for the equivalent circuit model parameters, and standard deviation, are given in the table in Fig. 4 . The various model parameters can be graphically interpreted from specific regions in the EIS measurements. At high frequencies the phase angle tends to zero and the modulus is solely due to $R_{\mathrm{S}}$. Similarly, at low frequencies the phase once again tends to zero, and the modulus represents the sum of $R_{\mathrm{S}}$ and $R_{\mathrm{ct}}$. In the mid-frequency range the phase tends to $-90^{\circ}$, representing a capacitive element, in this case the interface capacitance. The deviation of the model from experimental results at low frequencies can be attributed to the non-linear behavior of $R_{\mathrm{ct}}$ at low frequency (McAdams and Jossinet, 1994). As expected, the dendritically structured Pt black and TiN, with an increased total surface area, reduce the impedance modulus by approximately two orders of magnitude. The impedance of $900 \mu \mathrm{m}^{2}$ Pt black microelectrodes was found to be $4.84 \times 10^{4} \Omega$ at $1 \mathrm{kHz}$.

Cyclic voltammetry was used to determine the equilibrium exchange current density of bright Pt. The following measurement parameters were used: $5 \mathrm{mV}$ perturbation signal with respect to OCP, $0.5 \mathrm{mV} / \mathrm{s}$ scan rate, $0.15 \mathrm{mV}$ step potential, averaged over 10 scans. From the slope of the current-versus-applied-potential plot the exchange current density is calculated to be $8.5(10)^{8} \mathrm{~A} / \mathrm{cm}^{2}$ and $R_{\mathrm{ct}}$ is $3.0(10)^{5} 1 / 2$. This finding results in the omission of a Warburg impedance element in the model over the measurement frequency range used here.

The presented model is similar to others that have been previously applied, however here the parameters are associated with macroscopic physical processes contributing to the interface impedance (De Boer and Van Oosterom, 1978; Onaral and Schwan, 1982). Models that do attribute physical processes to circuit elements include a Warburg impedance element for diffusion (McAdams et al., 1995; Kovacs, 1994). In the frequency range used here no experimental evidence for a diffusional impedance has been found and it has therefore been omitted. It is possible that a diffusional impedance 

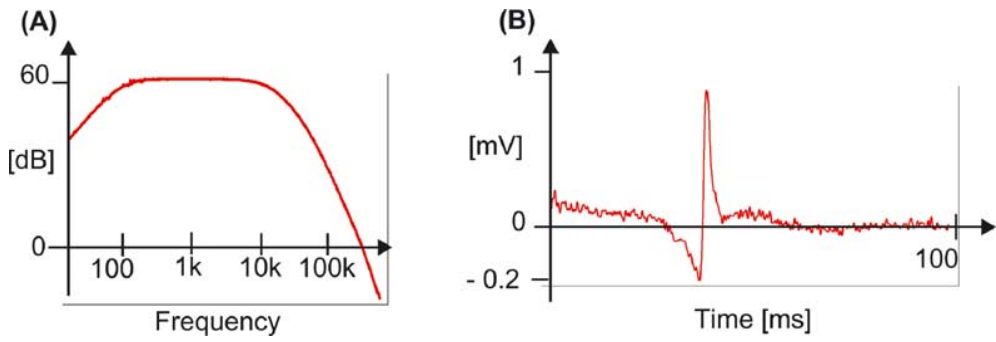

Fig. 8. Transfer function of the bandpass filter (A). Typical spike from embryonic chicken cardiomyocytes. High frequencies were cut-off at $3 \mathrm{kHz}$ (B).

would reveal itself at lower frequencies, which will be the subject of future investigations. Additionally, it is interesting to note that $n$, often termed the frequency dispersion coefficient, does not vary with surface roughness, as has been historically assumed (De Levie, 1965). The effect of the surface roughness on impedance has been the focus of intense research efforts and has resulted in many models such as the well known pore model of de Levie, models based on surface inhomogeneities leading to a distribution of relaxation times (Brug et al., 1984), and more recently in fractal geometry-based models (Nyikos and Pajkossy, 1985; Bates and $\mathrm{Chu}, 1992)$. The authors concur with the work of Pajkossy, where in the frequency range investigated the capacitance dispersion is attributed to adsorption effects (Pajkossy, 1994).

\subsection{Circuitry characterization}

Stimulation circuitry operation is demonstrated by generating a square-wave stimulus on-chip. The resulting signal on a selected electrode was measured using a probe tip. The bandpass filter has been electrically characterized, using a gain-phase analyzer (HP-4194A), with results shown in Fig. 8A. Corner frequencies were measured at $95 \pm 5 \mathrm{~Hz}$ and $72 \pm 10 \mathrm{kHz}$. The large error in the upper corner frequency was expected because it is due to the dominant pole of the operational amplifier of the first stage. A gain of $59.2 \pm 0.3 \mathrm{~dB}$ was measured at $1 \mathrm{kHz}$. The equivalent input noise of the filter is below $9 \mathrm{mV}_{\mathrm{RMS}}(100 \mathrm{~Hz}-100 \mathrm{kHz})$, as was measured with a spectrum analyzer (HP-4195A).

The performance of the temperature sensor was also measured. The temperature was swept from 20 to $50{ }^{\circ} \mathrm{C}$ and a 2-point calibration was performed. The measured sensitivity is about $210 \mathrm{mV} /{ }^{\circ} \mathrm{C}$ and the error is less than $\pm 0.2{ }^{\circ} \mathrm{C}$.

\subsection{First biological measurements}

First tests were performed with an in situ preparation of cardiomyocytes from fertilized chicken eggs at embryonic day 10. The hearts were extracted from the embryo, briefly rinsed with cold Dulbecco's PBS (Sigma D-8531, $\mathrm{Ca}^{2+}$ and $\mathrm{Mg}^{2+}$-free) and directly transferred onto the electrodes of the chip. A typical spike is shown in Fig. 8B. DC-offsets at the electrodes were measured to be up to $1 \mathrm{~V}$, which could cause saturation in the first amplification stage. Since the time-constant of the electrochemistry at the electrode-electrolyte interface is on the order of seconds, we used the on-chip stimulation circuitry to set the electrode to a working point.

\section{Conclusion}

An extracellular monolithic recording system, with 16 platinum electrodes, fabricated in industrial CMOS-technology combined with post-CMOS processing has been presented. The circuitry architecture is structured in a modular design where a repeatable circuitry unit comprises the signal transducing electrode, a bandpass filter for immediate signal conditioning, and a buffer for stimulation. The systems incorporates data converters and a digital control unit that scans the array and provides a digital interface to the outside world. Functionality of the circuitry components according to initial specifications has been verified. We showed that the chip is able to measure signals from electrogenic cells using embryonic chicken cardiomyocytes. Electrochemical impedance spectroscopy has been used to experimentally verify an equivalent circuit model of the electrode-electrolyte interface. The representation of the macroscopic physical processes occurring during the impedance measurement by the model parameters aids in the understanding of signal transduction, and may lead to an optimized transducer design.

\section{Acknowledgements}

The authors gratefully acknowledge funding from the Information Societies Technology (IST) European Union Future and Emerging Technologies program, contract number IST 2000-26045, and the Swiss Bundesamt fuer Bildung und Wissenschaft (BBW), contract number 00.0431.2. Iwan Schenker and Partick Schmutz provided invaluable help with impedance measurements and analysis.

\section{References}

Bai, Q., Wise, K.D., 2001. Single-unit neural recording with active microelectrode arrays. Trans. Biomed. Eng. 48 (8), 911-919. 
Bates, J.B., Chu, Y.T., 1992. Electrode-electrolyte interface impedance: experiments and model. Ann. Biomed. Eng. 20 (3), 349-362.

Berdondini, L., Overstolz, T., de-Rooij, N.F., Koudelka-Hep, M., Martinoia, S., Seitz, P., Wany, M., Blanc, N., 2002. High resolution electrophysiological activity imaging of in-vitro neuronal networks. In: Proceedings of the Second Annual International IEEE-EMBS Special Topic Conference on Microtechnologies in Medicine and Biology, Madison, WI, USA, pp. 241-244.

Borkholder, D.A., 1998. Cell based biosensors using microelectrodes. Stanford University.

Borkholder, D.A., Bao, J., Maluf, N.I., Perl, E.R., Kovacs, G.A., 1997. Microelectrode arrays for stimulation of neural slice preparations. J. Neurosci. 77, 61-66.

Brug, G.J., van den Eeden, A.L.G., Sluyters-Rehbach, M., Sluyters, J.H., 1984. The analysis of electrode impedances complicated by the presence of a constant phase element. J. Electroanal. Chem. 176, 275-295.

Bucher, V., Graf, M., Steizle, M., Nisch, W., 1999. Low-impedance thin-film polycrystalline silicon microelectrodes for extracellular stimulation and recording. Biosens. Bioelectron. 14 (7), 639-649.

Connolly, P., Clark, P., Curtis, A.S.G., Dow, J.A.T., Wilkinson, C.D.W., 1990. An extracellular microelectrode array for monitoring electrogenic cells in culture. Biosens. Bioelectron. 5, 223-234.

De Boer, R.W., Van Oosterom, A., 1978. Electrical properties of platinum electrodes: impedance measurements and time-domain analysis. Med. Biol. Eng. Comput. 16 (1), 1-10.

De Levie, R., 1965. The influence of surface roughness of solid electrode on electrochemical measurements. Electrochim. Acta 10, 113-130.

DeBusschere, B.D., Kovacs, G.T.A., 2001. Portable cell-based biosensor system using integrated CMOS cell-cartridges. Biosens. Bioelectron. 16 (7-8), 543-556.

Egert, U., Schlosshauer, B., Fennrich, S., Nisch, W., Fejtl, M., Knott, T., Muller, T., Hammerle, H., 1998. A novel organotypic long-term culture of the rat hippocampus on substrate-integrated multielectrode arrays. Brain Res. Protoc. 2 (4), 229-242.

Eversmann, B., Jenkner, M., Paulus C., Hofmann, F., Brederlow, R., Fromherz, P., Brenner, F., Schreiter, M., Gabl, R., Plehnert, K., Steinhauser, M., Eckstein, G., Schmitt-Landsiedel, D., Thewes, R., 2003. A $128 \times 128$ CMOS bio-sensor array for extracellular recording of neural activity. 2003 IEEE Int. Solid State Circuits Conf., 222-223.

Franks, W., Heer, F., McKay, I., Taschini, S., Sunier, R., Hagleitner, C., Hierlemann, A., Baltes, H., 2003. CMOS monolithic microelectrode array for stimulation and recording of natural neural networks. Transducers 2003, Boston, pp. 963-966.

Fricke, H., 1932. The theory of electrolytic polarization. Philos. Mag. 7 (14), 310-318

Gilchrist, K.H., Barker, V.N., Fletcher, L.E., DeBusschere, B.D., Ghanouni, P., Giovangrandi, L., Kovacs, G.T.A., 2001. General purpose, field-portable cell-based biosensor platform. Biosens. Bioelectron. 16, 557-564.

Grahame, D.C., 1952. Mathematical theory of the Faradaic admittance. J. Electrochem. Soc. 99 (12), 370C-385C.

Gross, G.W., 1979. Simultaneous single unit recording in vitro with a photoetched laser deinsulated gold multimicroelectrode surface. IEEE Trans. Biomed. Eng. BME-26 (5), 273-279.

Gross, G.W., Rhoades, B.K., Reust, D.L., Schwalm, F.U., 1993. Stimulation of monolayer networks in culture through thin-film indium-tin oxide recording electrodes. J. Neurosci. Methods 50 (2), 131-143.

Gross, G., Rhoades, B.K., Azzazy, H.M.E., Ming Chi, W., 1995. The use of neuronal networks on multielectrode arrays as biosensors. Biosens. Bioelectron. 10 (6-7), 553-567.

Heuschkel, M.O., Fejtl, M., Raggenbass, M., Bertrand, D., Renaud, P., 2002. A three-dimensional multi-electrode array for multi-site stimulation and recording in acute brain slices. J. Neurosci. Methods 114 (2), $135-148$.

Jenkner, M., Muller, B., Fromherz, P., 2001. Interfacing a silicon chip to pairs of snail neurons connected by electrical synapses. Biol. Cybern. 84 (4), 239-249.
Jimbo, Y., Robinson, H.P., 2000. Propagation of spontaneous synchronized activity in cortical slice cultures recorded by planar electrode arrays. Bioelectrochemistry 51 (2), 107-115.

Keefer, E.W., Gramowski, A., Gross, G.W., 2001a. nmDA receptor-dependent periodic oscillations in cultured spinal cord networks. J. Neurophysiol. 86, 3030-3042.

Keefer, E.W., Gramowski, A., Stenger, D.A., Pancrazio, J.J., Gross, G.W., 2001b. Characterization of acute neurotoxic effects of trimethylolpropane phosphate via neuronal network biosensors. Biosens. Bioelectron. 16 (7-8), 513-525.

Kovacs, G.T.A., 1994. Introduction to the theory, design, and modeling of thin-film microelectrodes for neural interfaces. In: Stenger, D.A., McKenna, T.M. (Eds.), Enabling Technologies for Cultured Neural Networks. Academic Press, London, pp. 121-165.

Kovacs, G.T.A., 2003. Electronic sensors with living cellular components. Proc. IEEE 91 (6), 915-929.

Maher, M.P., Pine, J., Wright, J., Yu Chong, T., 1999. The neurochip: a new multielectrode device for stimulating and recording from cultured neurons. J. Neurosci. Methods 87 (1), 45-56.

Marom, S., Shahaf, G., 2002. Development, learning and memory in large random networks of cortical neurons: lessons beyond anatomy Q. Rev. Biophys. 35 (1), 63-87.

McAdams, E.T., Jossinet, J., 1994. Physical interpretation of Schwan's limit voltage of linearity. Med. Biol. Eng. Comput. 32 (2), 126-130.

McAdams, E.T., Lackermeier, A., McLaughlin, J.A., Macken, D., Jossinet, J., 1995. The linear and non-linear electrical properties of the electrode-electrolyte interface. Biosens. Bioelectron. 10 (1-2), $67-74$

McFadden, P., 2002. Broadband biodetection: Holmes on a chip. Science 297, 2075-2976

Morefield, S.I., Keefer, E.W., Chapman, K.D., Gross, G.W., 2000. Drug evaluations using neuronal networks cultured on microelectrode arrays. Biosens. Bioelectron. 15 (7-8), 383-396.

Multi Channel Systems MCS, GmbH, Reutlingen, Germany.

Nyikos, L., Pajkossy, T., 1985. Fractal dimension and fractional power frequency-dependent impedance of blocking electrodes. Electrochim. Acta 30 (11), 1533-1540.

Onaral, B., Schwan, H.P., 1982. Linear and nonlinear properties of platinum electrode polarisation. I. Frequency dependence at very low frequencies. Med. Biol. Eng. Comput. 20 (3), 299-306.

Pajkossy, T., 1994. Impedance of rough electrodes. J. Electroanal. Chem. 364, 111-125.

Pancrazio, J.J., Bey Jr., P.P., Cuttino, D.S., Kusel, J.K., Borkholder, D.A., Shaffer, K.M., Kovacs, G.T.A., Stenger, D.A., 1998a. Portable cell-based biosensor system for toxin detection. Sens. Actuators B Chem. B53 (3), 179-185.

Pancrazio, J.J., Bey Jr., R.P., Loloee, A., Manne, S., Chao, H.C., Howard, L.L., Milton-Gosney, W., Borkholder, D.A., Kovacs, G.T.A., Manos, P., Cuttino, D.S., Stenger, D.A., 1998b. Description and demonstration of a CMOS amplifier-based-system with measurement and stimulation capability for bioelectrical signal transduction. Biosens. Bioelectron. 13 (9), 971-979.

Pancrazio, J.J., Whelan, J.P., Borkholder, D.A., Ma, W., Stenger, D.A., 1999. Development and application of cell-based biosensors. Ann. Biomed. Eng. 27, 687-711.

Pourbaix, M., 1974. Atlas of Electrochemical Equilibria in Aqueous Solutions. National Association of Corrosion Engineers, Brussels.

Prinz, A.A., Fromherz, P., 2000. Electrical synapses by guided growth of cultured neurons from the snail Lymnaea stagnalis. Biol. Cybernet. 82 (4), L1-L5.

Shahaf, G., Marom, S., 2001. Learning in networks of cortical neurons. J. Neurosci. 21 (22), 8782-8788.

Streit, J., Tscherter, A., Heuschkel, M.O., Renaud, P., 2001. The generation of rhythmic activity in dissociated cultures of rat spinal cord. Eur. J. Neurosci. 14 (2), 191-202.

Thiebaud, P., Beuret, C., Koudelka-Hep, M., Bove, M., Martinoia, S., Grattarola, M., Jahnsen, H., Rebaudo, R., Balestrino, M., Zimmer, J., 
Dupont, Y., 1999. An array of Pt-tip microelectrodes for extracellular monitoring of activity of brain slices. Biosens. Bioelectron. 14 (1), 61-65.

Thomas, C.A., Springer, P.A., Loeb, G.E., Berwald-Netter, Y., Okun, L.M., 1972. A miniature microelectrode array to monitor the bioelectric activity of cultured cells. Exp. Cell Res. 74, 61-66.

Tscherter, A., Heuschkel, M.O., Renaud, P., Streit, J., 2001. Spatiotemporal characterization of rhythmic activity in rat spinal cord slice cultures. Eur. J. Neurosci. 14 (2), 179-190. van der Schalie, W.H., Gardner, H.S., Bantle, J.A., de Rosa, C.T., Finch, R.A., Reif, J.S., Reuter, R.H., Backer, L.C., Burger, J., Folmar, L.C., Stokes, W.S., 1999. Animals as sentinels of human health hazards of environmental chemicals. Environ. Health Perspect. 107 (4), 309-315. Yeager, E., 1984. Electrocatalysts for $\mathrm{O}_{2}$ reduction. Electrochim. Acta 29 (11), 1527-1537.

Zeck, G., Fromherz, P., 2001. Noninvasive neuroelectronic interfacing with synaptically connected snail neurons immobilized on a semiconductor chip. Proc. Natl. Acad. Sci. U.S.A. 98 (18), 10457-10462. 